\title{
Study of Chiral Odd Generalized Parton Distributions at Jefferson Lab
}

\author{
Andrey Kim*t \\ University of Connecticut \\ E-mail: kenjo@jlab.org
}

\begin{abstract}
The quark-gluon dynamics manifests itself in a set of non-perturbative functions describing all possible spin-spin and spin-orbit correlations. The Transverse Momentum Dependent parton distributions (TMDs) and Generalized Parton Distributions (GPDs) carry information not only on the longitudinal but also on the transverse momentum and position of partons, providing rich and direct information on the orbital motion of quarks. Studies of the 3D PDFs are currently driving the upgrades of several existing facilities (JLab, COMPASS and RHIC), and the design and construction of new facilities worldwide (EIC, FAIR, and JPARC). Although the interest in GPDs and TMD PDFs has grown enormously, we are still in need of fresh theoretical and phenomenological ideas.

The main remaining challenges are extractions of actual 3D PDFs from different spin and azimuthal angle dependent distributions in a reliable and model independent way. In this talk, we present an overview of current status and future measurements of the 3D structure of the nucleon using exclusive and semi-inclusive production of photons and hadrons with the CLAS detector at Jefferson Lab.
\end{abstract}

XXVI International Workshop on Deep-Inelastic Scattering and Related Subjects (DIS2018) 16-20 April 2018

Kobe, Japan

\footnotetext{
* Speaker.

${ }^{\dagger}$ for CLAS Collaboration
} 
During the last few decades the Generalized Parton Distributions (GPDs) [1, 2, 3] formalism has emerged to describe the complicated spin structure of the nucleon and has demonstrated the ability to provide information for construction of multi-dimensional image of the nucleon.

At twist- 2 of the GPD framework, there are eight GPDs. Four of them, denoted by $H, \tilde{H}, E, \tilde{E}$, correspond to the parton helicity-conserving (chiral-even) processes, and the remaining four GPDs, denoted by $H_{T}, \tilde{H}_{T}, E_{T}, \tilde{E}_{T}$, correspond to parton helicity-flip (chiral-odd) processes. They depend on three kinematic variables: $x, \xi$ and $t$, where $x$ is the average parton longitudinal momentum fraction and $\xi$ (skewness) is half of the longitudinal momentum fraction transferred to the struck parton. The skewness can be expressed in terms of the Bjorken variable $x_{B}$ as $\xi \simeq x_{B} /\left(2-x_{B}\right)$, in which $x_{B}=Q^{2} /(2 p q), q$ is the four-momentum of the virtual photon and $Q^{2}=-q^{2}$. The momentum transfer to the nucleon is $t=\left(p-p^{\prime}\right)^{2}$, where $p$ and $p^{\prime}$ are the initial and final four momenta of the nucleon.

While chiral-even GPDs were studied extensively during last decades because most of the deeply virtual exclusive processes are sensitive to them, the chiral-odd GPDs are significantly less known. Their contribution is suppressed in the most of the reactions, and their parametrization in theoretical models are constrained by the moments obtained from Lattice QCD [4] and transversity PDF extracted from the analysis of azimuthal asymmetry in semi-inclusive deepl inelastic scattering [5]. Recently the measurements of exclusive $\pi^{0}$ and $\eta$ electroproduction structure functions $[6,7,8,9,10]$ has shown that Deeply Virtual Pseudoscalar Meson Production processes are particularly sensitive to chiral-odd GPDs $[11,12,13]$.

Early efforts to calculate $\pi^{0}$ electroproduction focused on chiral-even GPDs to parametrize the contributions from longitudinally polarized virtual photons but failed to describe the large cross section and beam spin asymmetry measurements of exclusive $\pi^{0}$ electroproduction [6, 14]. The inclusion of dominant chiral-odd components $[15,16,12,17,18]$ to calculate the contributions from transversely polarized virtual photons brought theoretical calculations into better agreement with experimental measurements [7, 8]. Both, experimental data and theoretical calculations show small $\sigma_{L T}$ and significantly larger $\sigma_{T T}$ as expected from the process dominated by transverse photon amplitudes.
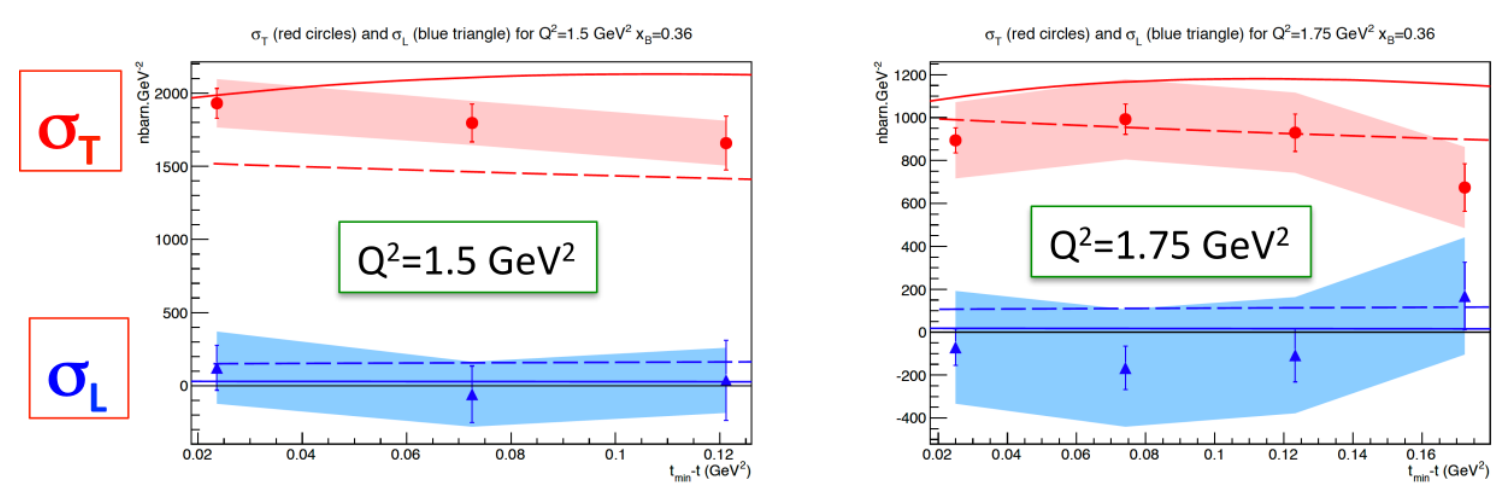

Figure 1: The Rosenbluth separation of the longitudinal and transverse contributions from deeply virtual $\pi^{0}$ electroproduction cross-section measurements [10].

Additionally, Fig. 1 recent analysis of the $\pi^{0}$ electroproduction cross section by Hall A Col- 
laboration at Jefferson Lab [10] used Rosenbluth technique to experimentally separate the contributions from longitudinal and transverse components and demonstrate the dominance of transverse virtual photon amplitudes. A fair agreement with models based on chiral-odd GPDs support the idea that pseudoscalar meson electroproduction offers the exciting opportunity to access chiral-odd GPDs experimentally.

Similarly to $\pi^{0}$ electroproduction, cross sections for $\eta$ meson production were measured from the same experimental dataset at Hall B, Jefferson Lab. Their structure functions were extracted and compared to the theoretical model calculations over the wide kinematic range and can be found in [9]. The comparison of the $\pi^{0}$ and preliminary $\eta$ structure functions is shown on Fig. 2. $\sigma_{U}$ drops by a factor of 2.5 for $\eta$-mesons in comparison with $\pi^{0}$ and $\sigma_{T T}$ drops by a factor of 10 . The chiral-odd GPD model [15, 16, 12] by Goloskokov and Kroll (curves) follows the experimental data. The inclusion of $\eta$ data into consideration and its successful description by theoretical model strengthens the statement about chiral-odd GPD dominance in the pseudoscalar electroproduction processes. Combined $\pi^{0}$ and $\eta$ data open the way for the flavor decomposition of the underlying transversity GPDs [19].
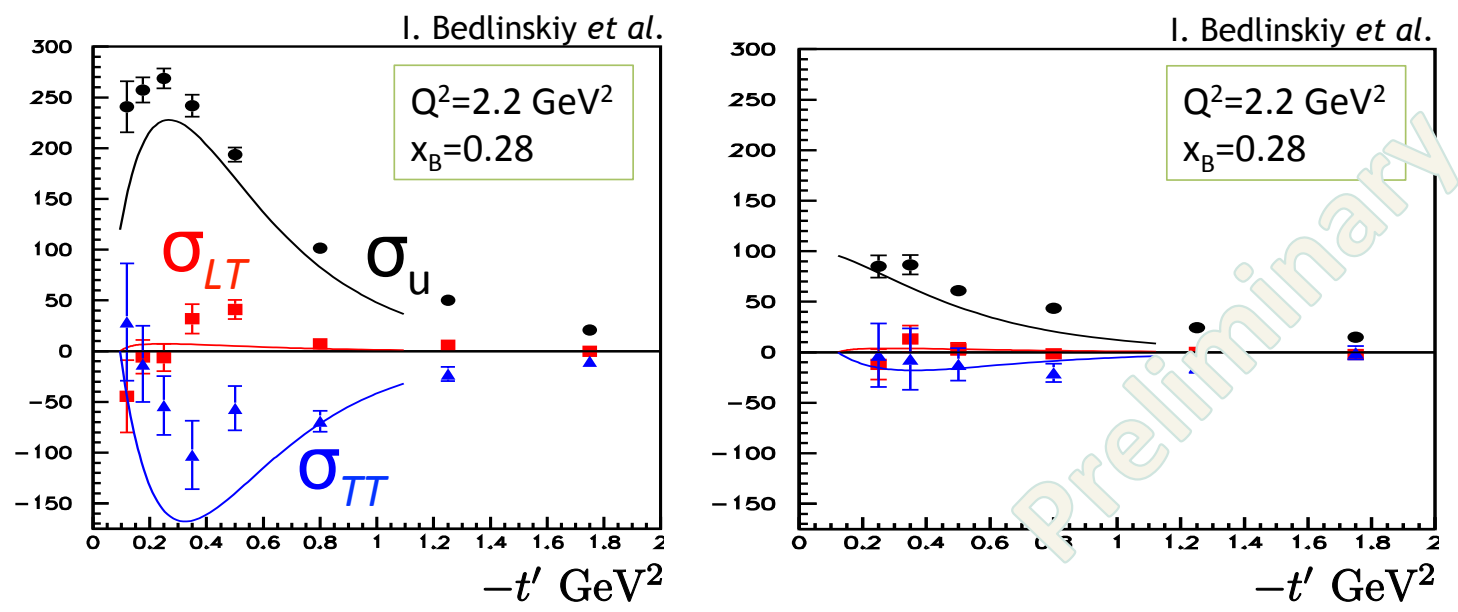

Figure 2: Structure function $\sigma_{T}+\varepsilon \sigma_{L}$ (black), $\sigma_{T T}$ (blue) and $\sigma_{L T}$ (red) as a function of $-t$ for $\pi^{0}$ (left) and $\eta$ (right) exclusive electroproduction for kinematic point $\left(Q^{2}=2.2 \mathrm{GeV}^{2}, x_{B}=0.28\right)$. Data points: CLAS results from [8] and [9]. Curves: theoretical predictions produced with GK handbag model.

The inclusion of beam, target and double spin asymmetries measurements for $\pi^{0}$ and $\eta$ electroproduction from Hall B, Jefferson Lab, [6, 20] and extraction of their azimuthal moments provide multiple experimental observables. Their combined analysis may allow separation of contributions from different underlying GPDs.

Within the Goloskokov-Kroll model the chiral-even contribution is small and pseudoscalar meson electroproduction is dominated by two contributing chiral-odd GPDs, $H_{T}$ and $\bar{E}_{T}$. From experiment we can access the convolutions of the chiral-odd GPD of the nucleon with the twist3 pion distribution amplitude, that we call generalized form factors (GFF). Combining different structure functions one can extract the contributions from GFF connected to specific GPD, as shown on Fig. 3. In this case $\sigma_{T}$ and $\sigma_{T T}$ were used to separate the contributions from $\left\langle H_{T}\right\rangle$ and $\left\langle\bar{E}_{T}\right\rangle$.

Furthermore, global analysis of these experimental measurements allow to perform the first attempt at quark flavor separation of chiral-odd GPDs. Comparing GFFs from $\pi^{0}$ and $\eta$ meson 

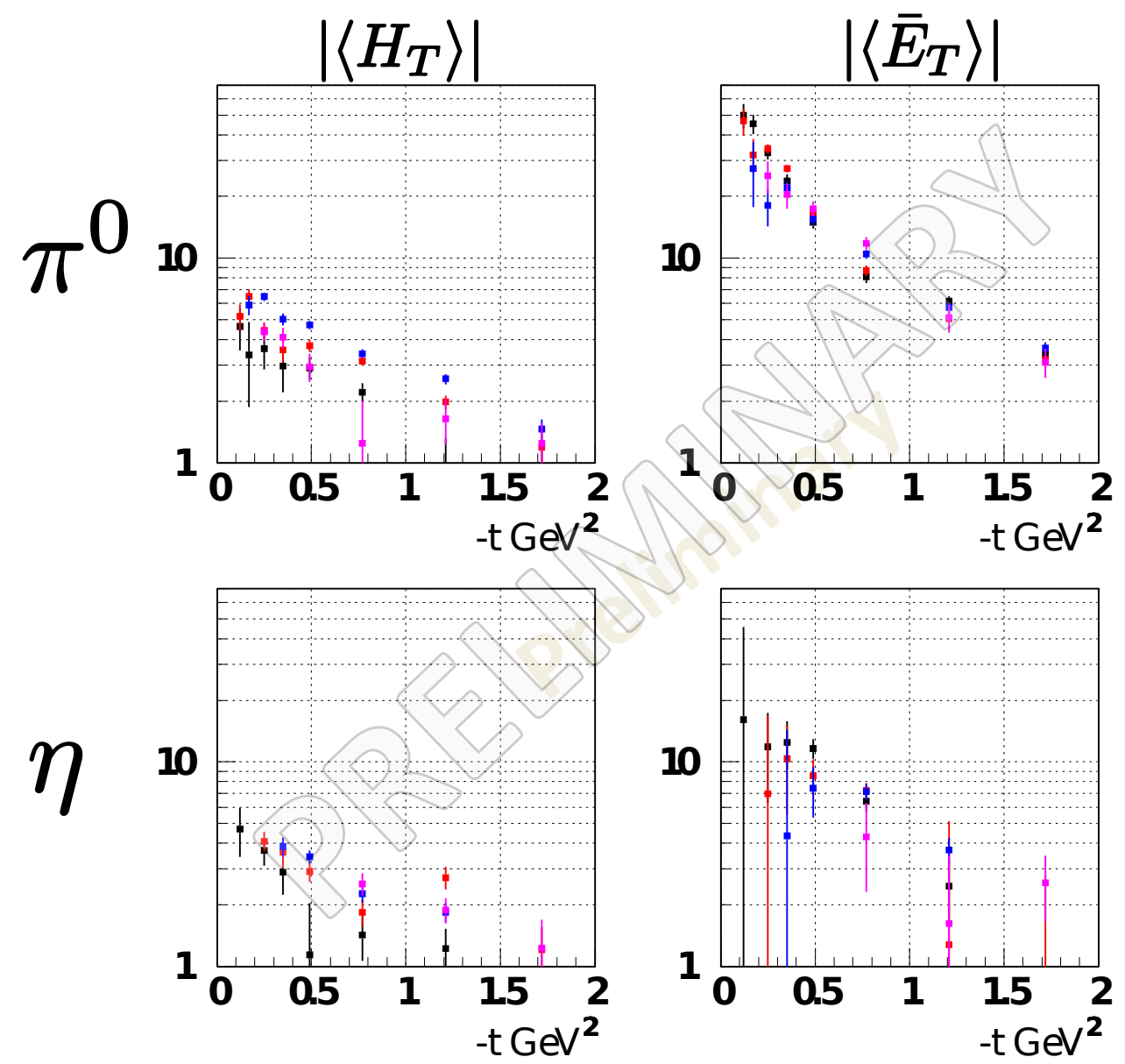

V. Kubarovsky, arXiv:1601.04367

Figure 3: Generalized form factors $\left\langle H_{T}\right\rangle$ and $\left\langle\bar{E}_{T}\right\rangle$ extracted using structure function measurements for $\pi^{0}$ and $\eta$ electroproduction [19].

electroproduction one can separate the contributions from different quarks. Assuming no contribution from strange quarks [12], in $\pi^{0}$ and $\eta$ electroproduction the GPD $F_{i}$ appears in the following combinations:

$$
\begin{aligned}
F_{i}^{\pi^{0}} & =\frac{1}{\sqrt{2}}\left[e_{u} F_{i}^{u}-e_{d} F_{i}^{d}\right] \\
F_{i}^{\eta} & =\frac{1}{\sqrt{6}}\left[e_{u} F_{i}^{u}+e_{d} F_{i}^{d}\right]
\end{aligned}
$$

Using equations above, we attempt to perform the flavor decomposition of generalized form factors based on the chiral-odd GPDs $H_{T}$ and $\bar{E}_{T}$, as shown on Fig. 4. The GFFs are complex variables, so an assumption on the relative phase between $u$ and $d$ GFFs was made to be either $0^{\circ}$ or $180^{\circ}$. As expected the $\left\langle\bar{E}_{T}\right\rangle$ for both $u$ and $d$ quarks had large values with the same sign, while $\left\langle H_{T}^{u}\right\rangle$ and $\left\langle H_{T}^{d}\right\rangle$ have opposite signs.

\section{Conclusion}

A large set of experimental observables for pseudoscalar meson electroproduction is available 

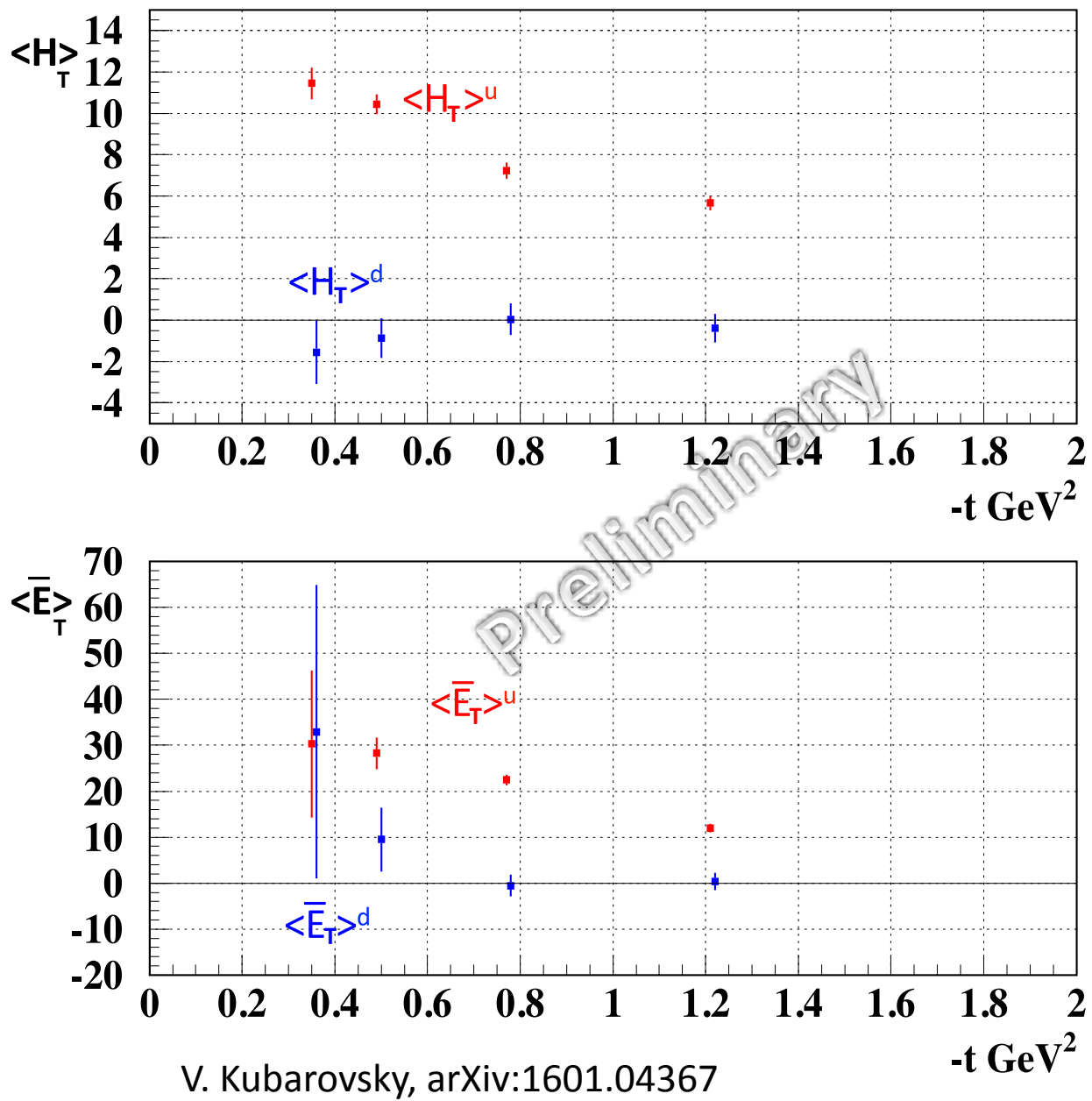

Figure 4: Preliminary. Top: extracted $\left\langle H_{T}\right\rangle^{u}$ (red) and $\left\langle H_{T}\right\rangle^{d}$ (blue), bottom: extracted $\left\langle\bar{E}_{T}\right\rangle^{u}$ (red) and $\left\langle\bar{E}_{T}\right\rangle^{d}$ (blue) as functions of $-t$.

from $6 \mathrm{GeV}$ era of measurements at Jefferson Lab. The successful description of unpolarized structure functions via inclusion of transverse virtual photon amplitudes offers an exciting opportunity to access elusive chiral-odd GPDs via deeply virtual meson production. Combined together, the variety of meson production channels allow to set a tighter constraints for the parametrization of the underlying GPDs perform their quark flavor separations.

The work presented here leads directly to the program of the Jefferson Lab $12 \mathrm{GeV}$ upgrade. The increased energy and luminosity will allow higher accuracy measurements over significantly wider kinematic range providing us with the opportunity to extend our analysis at higher $Q^{2}$ and test the mechanism of exclusive photon and meson electroproduction.

\section{Acknowledgements}

We acknowledge the outstanding efforts of the staff of the Accelerator and Physics Divisions at JLab. This work was supported in part by the U.S. Department of Energy and National Science Foundation, the French Centre National de la Recherche Scientifique and Commissariat à l'Energie 
Atomique, the Italian Istituto Nazionale di Fisica Nucleare, the National Research Foundation of Korea and the U.K. Engineering and Physical Science Research Council. Jefferson Science Associates (JSA) operates the Thomas Jefferson National Accelerator Facility for the United States Department of Energy under contract DE-AC05-06OR23177.

\section{References}

[1] X.D. Ji, Phys. Rev. Lett. 78, 610 (1997)

[2] A.V. Radyushkin, Phys. Lett. B380, 417 (1996)

[3] A.V. Belitsky, D. Mueller, A. Kirchner, Nucl. Phys. B629, 323 (2002)

[4] M. Göckeler, et al., Phys. Rev. Lett. 98, 222001 (2007)

[5] M. Anselmino, et al., Nucl. Phys. Proc. Suppl. 191, 98 (2009)

[6] R. De Masi, et al., Phys. Rev. C 77, 042201 (2008)

[7] I. Bedlinskiy, et al., Phys. Rev. Lett. 109, 112001 (2012)

[8] I. Bedlinskiy, et al., Phys. Rev. C90, 025205 (2014)

[9] I. Bedlinskiy, et al., Phys. Rev. C95, 035202 (2017)

[10] M. Defurne, et al., Phys. Rev. Lett. 117, 262001 (2016)

[11] S. Ahmad, G.R. Goldstein, S. Liuti, Phys. Rev. D79, 054014 (2009)

[12] S. Goloskokov, P. Kroll, Eur.Phys.J. A47, 112 (2011)

[13] G.R. Goldstein, J.O.G. Hernandez, S. Liuti, Phys. Rev. D 84, 034007 (2011)

[14] E. Fuchey, A. Camsonne, C. Munoz Camacho, M. Mazouz, G. Gavalian, et al., Phys.Rev. C83, $025201(2011)$

[15] S.V. Goloskokov, P. Kroll, Eur. Phys. J. C53, 367 (2008)

[16] S.V. Goloskokov, P. Kroll, Eur. Phys. J. C65, 137 (2010)

[17] J.O. Gonzalez-Hernandez, S. Liuti, G.R. Goldstein, K. Kathuria, Phys. Rev. C 88, 065206 (2013)

[18] G.R. Goldstein, J.O.G. Hernandez, S. Liuti, Phys. Rev. D 91, 114013 (2015)

[19] V. Kubarovsky, et al., arXiv 1601.04367 (2016)

[20] A. Kim, et al., Phys. Lett. B768, 168, (2017) 